\title{
Correlation of Myocardial Perfusion on Cardiac Magnetic Resonance Versus Myocardial Perfusion Scintigraphy in Cardiac Syndrome X
}

\author{
Ilse A.C. Vermeltfoort ${ }^{*}, 1$, Pieter G.H.M. Raijmakers ${ }^{1}$, Olga Bondarenko ${ }^{2}$, Anton Zwijnenburg ${ }^{3}$, \\ Mark B.M. Hofman ${ }^{4}$, Gerrit J.J. Teule ${ }^{5}$, Aernout M. Beek ${ }^{2}$ and Albert C. van Rossum ${ }^{2}$ \\ ${ }^{I}$ Department of Nuclear Medicine \& PET Research, VU University Medical Center, Amsterdam, The Netherlands \\ ${ }^{2}$ Department of Cardiology, VU University Medical Centre, Amsterdam, The Netherlands \\ ${ }^{3}$ Department of Nuclear Medicine, Spaarne Hospital, Hoofddorp, The Netherlands \\ ${ }^{4}$ Department of Physics and Medical Technology, VU University Medical Centre, Amsterdam, The Netherlands \\ ${ }^{5}$ Department of Nuclear Medicine, Maastricht University Medical Centre, Maastricht, The Netherlands
}

\begin{abstract}
Background: In cardiac syndrome X, which is a syndrome defined as chest pain, positive exercise stress testing and/or reversible myocardial perfusion defects during myocardial scintigraphy and normal coronary angiograms, the ischemic origin is still debated. No previous study compared the myocardial perfusion in stress first-pass cardiac magnetic resonance (CMR) versus stress single-photon emission computed tomography (SPECT) in cardiac syndrome X.

Methods: We performed stress SPECT and CMR imaging for 20 syndrome X patients. Perfusion analysis of the CMR was done by using the normalized upslope of myocardial signal enhancement to derive the myocardial perfusion index (MPI) and the myocardial perfusion reserve index (MPRI). The SPECT images were visually scored by 3 observers using a segmental model.

Results: An MPRI of $\leq 1.2$ was found for $31(9 \%)$ of the 335 segments, indicating local ischemia. SPECT indicated reversible perfusion defects for $39(12 \%)$ of the 335 segments. However, the combination of both an MPRI $\leq 1.2$ and a reversible perfusion defect was detected in only 3 segments.

Conclusions: Our data show about 10\% stress-induced myocardial perfusion abnormalities on CMR and SPECT, suggesting local ischemia. However, only in $1 \%$ of the segments there was concordance for the presence of myocardial ischemia with both exams. This result may be evidence for the variability over time of the mechanisms responsible for coronary microvascular dysfunction.
\end{abstract}

\section{INTRODUCTION}

About $20 \%$ of patients with anginal chest pain have normal coronary angiograms (CAG). The term cardiac "syndrome X" was introduced to describe these patients [1-3]. A subgroup of these patients has objective signs of ischaemia, such as the classic downsloping ST-segment depression on exercise ECG testing and/or a reversible defect detected by myocardial Single Photon Emission Computed Tomography (SPECT) [4-9].

The pathogenesis of this syndrome is far from clear. Different diagnostic imaging modalities, myocardial perfusion SPECT, cardiac Positron Emission Tomography (PET) and Cardiovascular Magnetic Resonance (CMR) imaging, have been used to try and reveal the physiological mechanism. Important results include reversible defects with myocardial SPECT and CMR, indicating local ischaemia [4-6, 8, 10]. Another important CMR finding is subendocardial ischaemia in a group of syndrome $\mathrm{X}$ patients. The ischaemia was located in the whole subendocardial layer of the left

\footnotetext{
*Address correspondence to this author at the Department of Nuclear Medicine \& PET Research, VU University Medical Centre, de Boelelaan 1117, 1007 MB, Amsterdam, The Netherlands; Tel: +31-20-4444214; Fax: +31-
} 20-4443090; E-mail: i.vermeltfoort@vumc.nl ventricle [11]. Subendocardial ischaemia may explain the reversible defects observed in other studies. However, the hypothesis of subendocardial ischaemia in cardiac syndrome $\mathrm{X}$ patients was not supported by our earlier CMR study done with patients having chest pain and normal coronary angiograms [12]. On the other hand, in that study the myocardial perfusion indexes (MPI) were calculated for the whole subendocardial and the whole subepicardial layer. Hence, regional ischaemia could have been undiscovered owing to average segmental MPI calculations as one mean subendocardial MPI and one mean subepicardial MPI.

To our knowledge the comparison of SPECT results and stress CMR results in cardiac syndrome $\mathrm{X}$ patients has not been done as yet. Therefore, we studied a group of patients with angina pectoris and normal coronary arteries using both SPECT and CMR to determine whether local reversible SPECT defects correlated with locally decreased myocardial perfusion by CMR.

\section{MATERIALS AND METHODOLOGY}

\section{Patient Characteristics and Inclusion/Exclusion Criteria}

All 20 patients had an established (1999-2004) diagnosis of classic syndrome $\mathrm{X}$, consisting of a typical history of 
exertional angina; an abnormal exercise electrocardiogram (0.1 $\mathrm{mV}$ horizontal or downsloping ST-segment depression of $80 \mathrm{msec}$ after the $\mathrm{J}$ point) and/or a reversible perfusion defect on a myocardial SPECT; and completely normal findings from coronary angiography (CAG), which was independently evaluated by two cardiologists in separate viewing sessions and without knowledge of the clinical information.

The exclusion criteria were: percutaneous transluminal coronary angioplasty (PTCA); coronary artery bypass grafting $(\mathrm{CABG})$ or prior myocardial infarction; spasm during the CAG reviewing; freedom from pain without medication; pregnancy; hypertension (defined as blood pressure over 140/90 mm Hg); diabetes (defined by a fasting glucose level above $7.8 \mathrm{mmol}$ per litre or a random-sample glucose level above $11.1 \mathrm{mmol}$ per litre); arrhythmias such as paroxysmal atrial fibrillation (PAF); left bundle branch block; valve dysfunction (other than mitral valve insufficiency grade 1); and abnormal left ventricular ejection fraction (LVEF $<50$ $\%$ ) or other structural abnormalities of the heart. Furthermore, patients having general contra-indications for CMR imaging according to the MR safe practice guidelines were also excluded [13]. Finally, none of the patients had electrocardiographic signs of left ventricular hypertrophy (defined as a value above $35 \mathrm{~mm}$ for the sum of the height of the $\mathrm{S}$ wave in lead $V_{1}$ and the height of the $R$ wave in lead $V_{5}$ ), as later confirmed by CMR. None of the patients had any change in clinical condition or medication between the investigations.

Five patients had abnormal exercise electrocardiograms suggesting ischaemia. Eight patients showed an inconclusive exercise ECG test (chest pain without significant STsegment depression).

The patients received calcium-channel blockers ( 7 patients), nitrates (4 patients), beta-blockers (6 patients), ACE inhibitors ( 1 patient), or no treatment ( 5 patients). These numbers reflect the fact that some patients received a combination of these drugs.

The study complied with the Declaration of Helsinki, was approved by the institutional ethics committee, and all patients gave written informed consent.

\section{Myocardial Perfusion Spect (Stress-Rest 2 Days Protocol)}

Patients were instructed to stop all cardiac medication for 2 days. The exercise was performed on a calibrated ergometer using a symptom-limited test with stepwise increased work. A 12-lead electrocardiogram and blood pressure were monitored throughout the duration. SPECT imaging was performed 45-60 minutes after peak exercise radiotracer injection. Day two of this study was allocated to rest. The data acquisition parameters were identical for the rest and stress studies.

Bull's-eye generation with a visual analysis using a 19segmental model was performed. Only 18 segments were used for comparison with the CMR. The apical segment was excluded because no myocardial perfusion was measured with CMR in the apical segment of the left ventricle.

All SPECT results were interpreted by three experienced observers, with any disagreements being resolved by consensus. This consensus method was used to avoid subjective individual SPECT results. These observers had no access to the results of the CMR study. Perfusion of both the stress and rest study was graded on a scale of 0 to 4 , with 0 representing normal perfusion and 4 representing a very severe perfusion defect.

\section{CMR Scan Protocol (First Pass Stress-Rest)}

The patients were instructed to stop all cardiac medications and refrain from caffeine-containing beverages 24 hours before CMR imaging, and to eat a light breakfast on the day of the test. Patients receiving beta-blocking drugs stopped this medication for at least three drug half-lifetimes. Before testing, an intravenous line of normal saline solution, with a 20-gauge canula, was positioned in the antecubital veins in both arms. We used a single canula for administration of contrast and a separate canula for the administration of adenosine.

Imaging was performed with a $1.5 \mathrm{~T}$ whole body MRI scanner (Magnetom Sonata, Siemens, Erlangen, Germany) using a four-element phased array cardiac receiver coil, and with the patient in a supine position. Scout images were acquired in the long axis and short axis orientations in order to specify the final short axis views.

To obtain the first pass contrast-enhanced images, a saturation prepared single shot spoiled gradient-echo pulse sequence, was applied (repetition time $2.0 \mathrm{~ms}$, echo time 1.0 ms, flip angle $12^{\circ}$, receiver band with $770 \mathrm{~Hz} /$ pixel, saturation delay $120 \mathrm{~ms}$ ). The spatial resolution was $3-3.3 \times 2.3-$ $2.7 \times 8 \mathrm{~mm}^{3}$, with an image matrix of $128 \times 73-93$.

Perfusion scans were performed during the last minute of a 4-minute adenosine infusion $(140 \mu \mathrm{g} / \mathrm{kg} / \mathrm{min})$, and at rest 15 minutes later. Three short axis slices from apex to base at $25 \%, 50 \%$ and $75 \%$ of the end-systolic ventricular length were imaged. Both stress- and rest-perfusion images were acquired during breath-holding for 50 heartbeats and during the first pass of $0.05 \mathrm{mmol} / \mathrm{kg}$ gadolinium-based contrast agent (Magnevist, Schering AG, Berlin, Germany), flushed with $15 \mathrm{ml}$ of $0.9 \% \mathrm{NaCl}$ (flow rate $5 \mathrm{ml} / \mathrm{s}$; Medrad, Spectris).

During the waiting period between the stress- and restperfusion scans, ECG-gated cine images were acquired using a breath-hold segmented balanced steady-state free precession sequence (bSSFP). Eight to ten short-axis views were obtained every $10 \mathrm{~mm}$, starting from the mitral valve insertion and covering the entire left ventricle. The cine bSSFP sequence parameters were a temporal resolution of $47 \mathrm{~ms}$, excitation angle of $60^{\circ}$, receiver bandwidth $930 \mathrm{~Hz} /$ pixel, $\mathrm{TR} / \mathrm{TE}$ of 3.1/1.6 ms, matrix $256 \times 138-161$ and voxel size of $1.3-1.4 \times 1.8-2.0 \times 5.0-6.0 \mathrm{~mm}^{3}$.

To exclude myocardial scar tissue, late contrast-enhanced images were acquired 10 minutes after the last contrast injection and in the same orientation as the first pass contrastenhanced images, using a 2D segmented inversion recovery spoiled gradient-echo pulse sequence triggered to enddiastole (repetition time/echo time $=9.6 / 4.4 \mathrm{~ms}$, flip angle $25^{\circ}$, number of excitations $=1$, matrix $208 \times 256$, typical voxel size of $1.6 \times 1.3 \times 5.0 \mathrm{~mm}^{3}$, receiver bandwidth 130 $\mathrm{Hz} /$ pixel). The inversion time was set to null the signal of normal myocardium was typically in the range of 220-290 ms. 


\section{MRI Analysis}

An 18-segment model was used, dividing the left ventricle into six basal, six midventricular, and six distal segments. The CMR analysis was performed separately from the SPECT analysis, and the CMR observer had no access to the results of the SPECT study and other diagnostic procedures. Delayed contrast-enhanced images were assessed for the presence of any hyper-enhancement.

Semi-quantitative analysis was done using a dedicated software package (Mass 5.0, Medis, Leiden, Netherlands). The endocardial and epicardial contours on perfusion images were traced, and corrected manually for cardiac motion. Each slice was divided into six equiangular segments, starting from the inferior septal insertion of the right ventricle. These segments were further subdivided into subendocardial and subepicardial regions, which were traced with their outer borders close to the endocardial and epicardial surfaces, and inner borders adjacent to each other in the mid-wall. To obtain information about the input function, an additional region was drawn in the left ventricular cavity.

Curves showing relative signal intensity versus time were generated for each of the defined regions. The maximum upslopes of the myocardium and the left ventricular blood pool were determined using 5- and 3-point linear fits, respectively. The results for the myocardial regions were corrected for differences in the arterial input function of the contrast agent bolus by dividing the myocardial upslope by the left ventricular blood pool upslope [14]. An index for myocardial perfusion reserve (MPRI) was calculated by dividing the MPI's at maximal vasodilatation by the values at rest. Segments with MPRI $\leq 1.2$ were considered to indicate ischaemia, as in the work of Ibrahim [15].

\section{Statistics}

Following the SPECT segment scheme (but using only 18 instead of 19 segments, as mentioned earlier) we classified the CMR segments as non-ischaemic and ischaemic. Mean MPRI results were obtained for the transmural, subendocardial and subepicardial regions. The summary values are presented as means \pm SD. Differences between the MPRI transmural, subendocardial and subepicardial non-ischaemic and ischaemic segments were tested using an unpaired student test whereby a $p$ value of $<0.05$ was considered significant.

\section{RESULTS}

\section{Heart Rates, Blood Pressure and Chest Pain}

The baseline heart rate was $71 \pm 11 \mathrm{BPM}$, increasing to $94 \pm 14$ BPM during adenosine infusion. The baseline blood pressure was systolic $139 \pm 20 \mathrm{~mm} \mathrm{Hg}$ and diastolic $80 \pm 9$ $\mathrm{mm} \mathrm{Hg}$. During maximum vasodilatation the blood pressure was systolic $140 \pm 18 \mathrm{~mm} \mathrm{Hg}$ and diastolic $73 \pm 10 \mathrm{~mm} \mathrm{Hg}$. Furthermore, 14 of the 20 patients experienced severe chest pain during adenosine infusion. The remaining 6 patients did not experience any chest pain.

\section{SPECT Visual Analysis}

The SPECT results showed reversible perfusion defects in 16 patients, a mild fixed defect in two patients, and one reversed perfusion pattern and one normal perfusion in the remaining two patients. The reversible perfusion defects were found in $47(12 \%)$ of the 360 segments corresponding to all 20 syndrome $\mathrm{X}$ patients (Fig. 1).

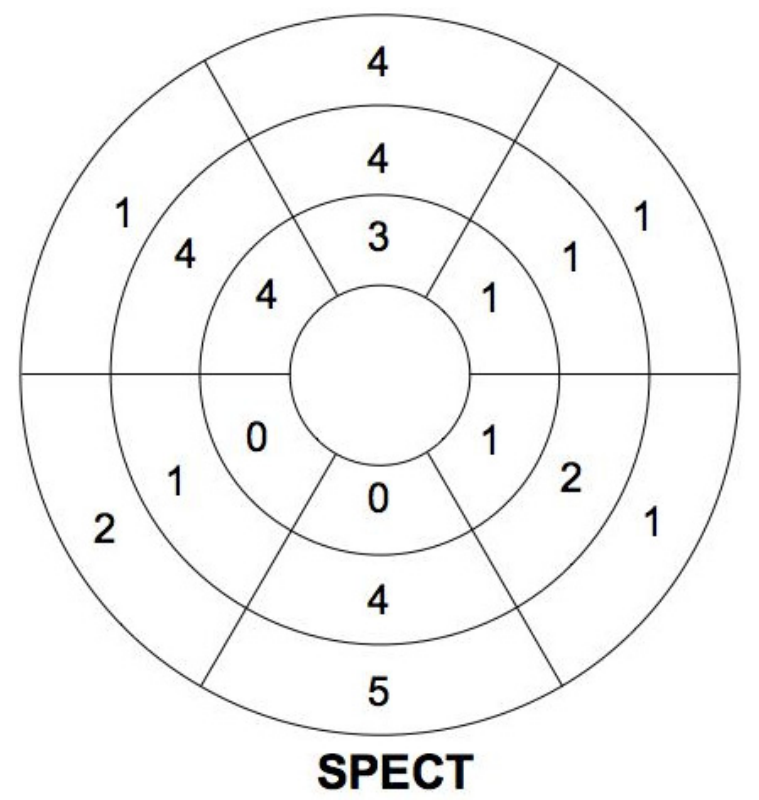

Fig. (1). Localisation and total number of reversible perfusion defect segments in 20 syndrome $\mathrm{X}$ patients.

\section{CMR Analysis}

No hyper-enhancement was seen on the late contrastenhanced images. A reliable myocardial perfusion index (MPI) during stress and rest could be measured for 335 (93\%) of the 360 segments. MPI measurements for $25(7 \%)$ of the segments were impossible owing to left ventricle outflow tract artefacts.

An index for myocardial perfusion reserve (MPRI) was calculated as the ratio of the MPI during stress to the MPI at rest. $31(9.3 \%)$ of the 335 segments were assigned a transmural MPRI $\leq 1.2$, indicating local ischaemia (Fig. 2).

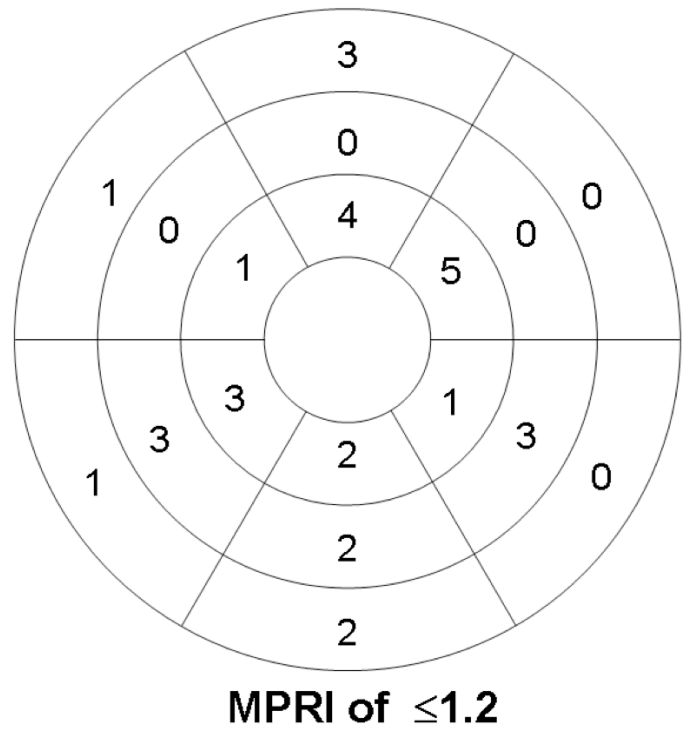

Fig. (2). Localisation and total number of segments with a transmural Myocardial Perfusion Reserve Index (MPRI) $\leq 1.2$ in 20 syndrome X patients. 


\section{Comparison of SPECT and CMR}

Of a total of 4 reversible segments no reliable MPRI could be analysed, as it was planned to contain major parts of the left ventricular outflow tract (basal slice anterior and anterolateral). In 39 of the 335 segments also imaged by CMR (12\%), a reversible perfusion defect was found with SPECT

Only 3 segments gave a matched result of a reversible perfusion defect and a transmural MPRI $\leq 1.2$. 28 segments had a normal SPECT result combined with a low transmural MPRI ( $\leq 1.2)$ (mismatch); and 36 segments had an ischaemic SPECT result combined with a normal CMR (mismatch).

The summed bull's-eye of SPECT and CMR studies of these 20 patients show a similar distribution of ischaemic segments throughout the left ventricle, and there was no specific vascular territory with a high number of ischaemic segments. Analysis showed there was a lack of spatial correlation between the SPECT reversible perfusion defect segments and the MPRI ischaemic segments in each patient.

The mean transmural MPRI was the same in the normal and ischaemic SPECT segments $1.85 \pm 0.65$ versus $1.85 \pm$ $0.58(p=1)$. Fig. (3) shows that the mean subendocardial MPRI values were not different in the normal and ischaemic SPECT segments $(1.68 \pm 0.54$ versus $1.70 \pm 0.51, \mathrm{p}=$ 0.860). Also, the mean subepicardial MPRI was similar in the normal SPECT segments versus ischaemic segments 2.01 \pm 0.89 versus $2.00 \pm 0.74(\mathrm{p}=0.882)$.

\section{subendocardial MPR}

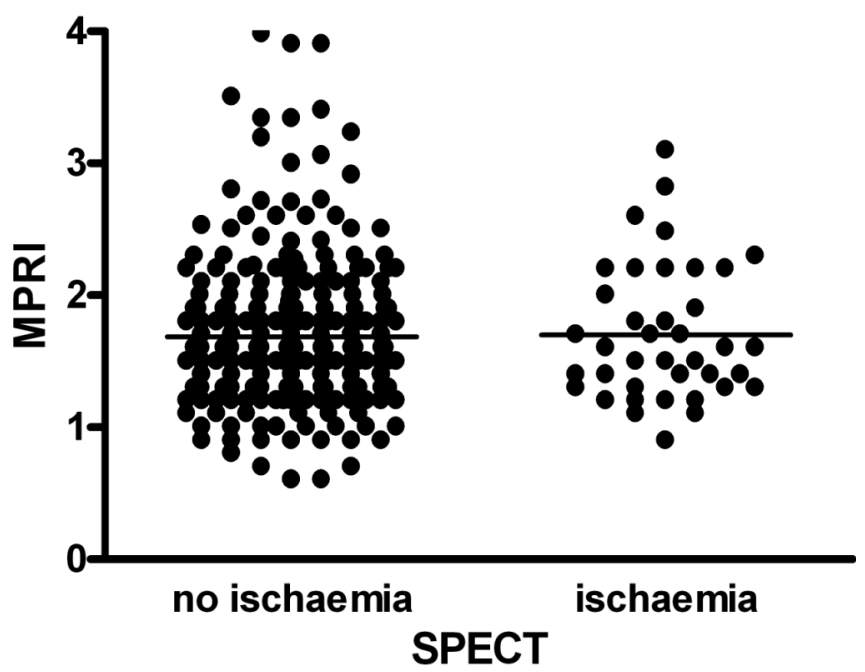

Fig. (3). Mean subendocardial MPRI in the normal and ischaemic SPECT segments, SPECT $=$ single-photon emission computed tomography.

\section{DISCUSSION}

We found evidence for local ischaemia in $10 \%$ of the left ventricle area with both the CMR study and the SPECT study. However, there was no spatial match for ischaemia detected by SPECT and CMR for these CSX patients. Only 3 segments out of 335 (1\%) were concordant in both exams. Mismatches were present in 64 segments, mutually revealing ischaemia by one technique and a normal result by the other technique. Furthermore, SPECT-derived normal and ischaemic segments had similar mean myocardial perfusion reserve indexes (MPRI). In the light of these results, we conclude that for this group of patients local ischaemia was unlikely to be present in a fixed area of the left ventricle, Furthermore, it is unlikely that in this group of patients ischaemia was caused by a fixed anatomically or functionally abnormal vessel. On the other hand, the local ischaemia found with two independent techniques at different times could be explained by transient ischaemia. Local ischaemia might be present in a patient at different locations over time, a phenomenon that we speculatively describe as migrating ischaemia.

These results do not necessarily conflict with our earlier analysis, in which CMR detected significant increases of the myocardial perfusion both in the subendocardial and subepicardial layer [12]. Calculation of an average MPI for the whole subendocardial layer may mask local ischaemia in smaller segmental area's. Segmental analysis of the MPI may be more accurate than calculations of whole subendo- or subepicardial layers, especially since these layers will receive blood supply from different coronary vessels.

To our knowledge this is the first study directly comparing myocardial perfusion by stress SPECT and CMR imaging on a segmental basis for the same cardiac syndrome $\mathrm{X}$ patients in the same territories. Our transient ischaemic findings may be consistent with the arguments of Maseri et al. [16]. These investigators speculated that distal to the most constricted microvessels there could be a patchy distribution of small foci of ischaemia which, when confluent, might be sufficient to cause transient ST-segment depression and also myocardial perfusion abnormalities [16]. Some support for this view is provided by a study by Osamichi et al. using PET and histopathology. They showed extensive myocardial ${ }^{18}$ F-fluorodeoxyglucose (FDG) uptake in combination with irregular narrowing of the coronary microvessels at the level of capillaries as well as meta-arterioles in syndrome $\mathrm{X}$ patients [17]. Such a patchy distribution of microvascular dysfunction might be responsible for the segmental SPECT and CMR abnormalities in the present study, hence suggesting microvascular migrating or transient ischaemia in cardiac syndrome $\mathrm{X}$ patients.

Our study is apparently partly at variance with the recent study by Lanza et al. in which they found a relation between subendocardial perfusion defects with CMR and a reduced coronary flow reserve by coronary doppler in the same (anterior) region [10]. However, no quantitative CMR analysis was performed. On the other hand, Lanza study found reduced coronary flow reserve in the LAD coronary artery in 2 patients $(11 \%)$ who displayed reversible perfusion defects with CMR only in the right coronary artery territory. This suggests a variability over time of the mechanisms responsible for the coronary microvascular dysfunction [10], which is in line with our study, although in our population it is more frequently occurring.

Variations in the SPECT and CMR measurements in the present study may have contributed to the differences we 
found between the results of the two measurement techniques. This is despite our efforts to reduce the likelihood of variations and false positive results by using a consensus reading for the SPECT studies and a strict cut-off level for the MPRI calculations. For example, MPRI of less than 1.2 indicates a clearly limited increase of myocardial perfusion, which is compatible with a more than $75 \%$ obstruction of coronary flow [18]. Another potential limitation of the present study is the use of two different forms of stress testing for CMR and SPECT. However, for detection of ischaemia both types of stress testings give good results. An additional explanation for mismatched non-ischaemic and ischaemic segments emerges from necessarily using the two techniques at different times. There were, however, no changes in cardiac medication, complaints, or changes in possible confounding factors like smoking. Furthermore, reversibility of anatomical changes of epicardial vessels is rather unlikely in patients with angina pectoris.

With regard to the techniques themselves, we assumed that CMR would be able to detect more ischaemia missed by SPECT owing to the higher spatial resolution of CMR [19]. Our results do not support this assumption, since we found a comparable percentage of ischaemic segments with both techniques. The accuracy of quantitative first-pass perfusion CMR imaging using adenosine-induced hyperaemia has been demonstrated in several recent studies [20,21], and nuclear myocardial perfusion imaging with SPECT is widely accepted as a standard evaluation of myocardial perfusion. However, our comparison of CMR with SPECT may not have been optimal. For example, the apical segment was not assessed by CMR and was excluded from this comparison. Thus our study lacks a direct comparison of CMR and SPECT in the apex. Also, the segments used by the segmental SPECT model were somewhat larger than the segments used in the CMR analysis. Nevertheless, it is unlikely that the above-mentioned differences explain the $95 \%$ mismatch of ischaemic segments. Furthermore, studies state that first pass CMR and myocardial perfusion SPECT correlate well and demonstrate fair-to-good agreement $(70-90 \%)$ in the assessment of perfusion defects [22-25].

\section{CONCLUSIONS}

In conclusion, our data show about $10 \%$ stress-induced myocardial perfusion abnormalities on CMR and SPECT, suggesting local ischaemia in cardiac syndrome $\mathrm{X}$ patients. We found no evidence for ischaemia in fixed areas of the left ventricle in syndrome $X$. We speculate that our results may be compatible and explicable with a coronary microvascular dysfunction that is variable over time, as recently stated by Lanza [10]. Further studies are necessary to confirm this hypothesis.

\section{ACKNOWLEDGEMENTS}

O. Bondarenko is supported by the Netherlands Heart Foundation, grant number 2001B158.

Thanks to Dr. R.J.H. Wanhill for his critical review of the manuscript. Thanks to D.A.M Odekerken and A.F.M. Kuijper PhD for collecting the patients.

\section{REFERENCES}

[1] Kemp HG, Jr. Left ventricular function in patients with the anginal syndrome and normal coronary arteriograms. Am J Cardiol 1973; 32(3): 375-6.

[2] Kemp HG, Jr, Vokonas PS, Cohn PF, Gorlin R. The anginal syndrome associated with normal coronary arteriograms. Report of a six year experience. Am J Med 1973; 54(6): 735-42.

[3] Likoff W, Segal BL, Kasparian H. Paradox of normal selective coronary arteriograms in patients considered to have unmistakable coronary heart disease. N Engl J Med 1967; 276(19): 1063-6.

[4] Fragasso G, Rossetti E, Dosio F, et al. High prevalence of the thallium-201 reverse redistribution phenomenon in patients with syndrome X. Eur Heart J 1996; 17(10): 1482-7.

[5] Kao CH, Wang SJ, Ting CT, Chen YT. Thallium-201 myocardial SPET in strictly defined syndrome X. Nucl Med Commun 1995; 16(8): 640-6.

[6] Kao CH, Wang SJ, Ting CT, Chen YT. Tc-99m sestamibi myocardial SPECT in syndrome X. Clin Nucl Med 1996; 21(4): 280-3.

[7] Kaul S, Newell JB, Chesler DA, et al. Quantitative thallium imaging findings in patients with normal coronary angiographic findings and in clinically normal subjects. Am J Cardiol 1986; 57(8): 50912.

[8] Tweddel AC, Martin W, Hutton I. Thallium scans in syndrome X. Br Heart J 1992; 68(1): 48-50.

[9] Lanza GA. Cardiac syndrome X: a critical overview and future perspectives. Heart 2007; 93(2): 159-66.

[10] Lanza GA, Buffon A, Sestito A, et al. Relation between stress-induced myocardial perfusion defects on cardiovascular magnetic resonance and coronary microvascular dysfunction in patients with cardiac syndrome X. J Am Coll Cardiol 2008; 51(4): 466-72.

[11] Panting JR, Gatehouse PD, Yang GZ, et al. Abnormal subendocardial perfusion in cardiac syndrome $\mathrm{X}$ detected by cardiovascular magnetic resonance imaging. N Engl J Med 2002; 346(25): 194853.

[12] Vermeltfoort IA, Bondarenko O, Raijmakers PG, et al. Is subendocardial ischaemia present in patients with chest pain and normal coronary angiograms? A cardiovascular MR study. Eur Heart J 2007; 28(13): 1554-8.

[13] Kanal E, Borgstede JP, Barkovich AJ, et al. American College of Radiology White Paper on MR Safety. AJR Am J Roentgenol 2002; 178(6): 1335-47.

[14] Nagel E, Klein C, Paetsch I, et al. Magnetic resonance perfusion measurements for the noninvasive detection of coronary artery disease. Circulation 2003; 108(4): 432-7.

[15] Ibrahim T, Nekolla SG, Schreiber K, et al. Assessment of coronary flow reserve: comparison between contrast-enhanced magnetic resonance imaging and positron emission tomography. J Am Coll Cardiol 2002; 39(5): 864-70

[16] Maseri A, Crea F, Kaski JC, Crake T. Mechanisms of angina pectoris in syndrome X. J Am Coll Cardiol 1991; 17(2): 499-506.

[17] Osamichi S, Kouji K, Yoshimaro I, et al. Myocardial glucose metabolism assessed by positron emission tomography and the histopathologic findings of microvessels in syndrome X. Circ J 2004; 68(3): 220-6.

[18] Ibrahim T, Nekolla SG, Schreiber K, et al. Assessment of coronary flow reserve: comparison between contrast-enhanced magnetic resonance imaging and positron emission tomography. J Am Coll Cardiol 2002; 39(5): 864-70.

[19] Wagner A, Mahrholdt H, Sechtem U, Kim RJ, Judd RM. MR imaging of myocardial perfusion and viability. Magn Reson Imaging Clin N Am 2003; 11(1): 49-66.

[20] Al Saadi N, Nagel E, Gross M, et al. Noninvasive detection of myocardial ischemia from perfusion reserve based on cardiovascular magnetic resonance. Circulation 2000; 101(12): 1379-83.

[21] Nagel E, Klein C, Paetsch I, et al. Magnetic resonance perfusion measurements for the noninvasive detection of coronary artery disease. Circulation 2003; 108(4): 432-7.

[22] Keijer JT, van Rossum AC, van Eenige MJ, et al. Magnetic resonance imaging of regional myocardial perfusion in patients with single-vessel coronary artery disease: quantitative comparison with (201)Thallium-SPECT and coronary angiography. J Magn Reson Imaging 2000; 11(6): 607-15. 
[23] Matheijssen NA, Louwerenburg HW, van Rugge FP, et al. Comparison of ultrafast dipyridamole magnetic resonance imaging with dipyridamole SestaMIBI SPECT for detection of perfusion abnormalities in patients with one-vessel coronary artery disease: assessment by quantitative model fitting. Magn Reson Med 1996; 35(2): 221-8.

[24] Fenchel M, Helber U, Simonetti OP, et al. Multislice first-pass myocardial perfusion imaging: Comparison of saturation recovery
(SR)-TrueFISP-two-dimensional (2D) and SR-TurboFLASH-2D pulse sequences. J Magn Reson Imaging 2004; 19(5): 555-63.

[25] Thiele H, Plein S, Breeuwer M, et al. Color-encoded semiautomatic analysis of multi-slice first-pass magnetic resonance perfusion: comparison to tetrofosmin single photon emission computed tomography perfusion and X-ray angiography. Int $\mathrm{J}$ Cardiovasc Imaging 2004; 20(5): 371-84.

(C) Vermeltfoort et al.; Licensee Bentham Open.

This is an open access article licensed under the terms of the Creative Commons Attribution Non-Commercial License (http: //creativecommons.org/licenses/by-nc/ $3.0 /$ ) which permits unrestricted, non-commercial use, distribution and reproduction in any medium, provided the work is properly cited. 